\title{
Adaptive Mixture Modeling Metropolis Methods for Bayesian Analysis of Nonlinear State-Space Models
}

\begin{abstract}
Jarad NIEMI and Mike WeST
We describe a strategy for Markov chain Monte Carlo analysis of nonlinear, nonGaussian state-space models involving batch analysis for inference on dynamic, latent state variables and fixed model parameters. The key innovation is a MetropolisHastings method for the time series of state variables based on sequential approximation of filtering and smoothing densities using normal mixtures. These mixtures are propagated through the nonlinearities using an accurate, local mixture approximation method, and we use a regenerating procedure to deal with potential degeneracy of mixture components. This provides accurate, direct approximations to sequential filtering and retrospective smoothing distributions, and hence a useful construction of global Metropolis proposal distributions for simulation of posteriors for the set of states. This analysis is embedded within a Gibbs sampler to include uncertain fixed parameters. We give an example motivated by an application in systems biology. Supplemental materials provide an example based on a stochastic volatility model as well as MATLAB code.
\end{abstract}

Key Words: Bayesian computation; Forward filtering, backward sampling; Regenerating mixture procedure; Smoothing in state-space models; Systems biology.

\section{INTRODUCTION}

Motivated by problems of fitting and assessing structured, nonlinear dynamic models to time series data arising in studies of cellular networks in systems biology, we revisit the problem of Bayesian inference on latent, time-evolving states and fixed model parameters in a state-space model context. In recent years, this general area has seen development of a number of customized Monte Carlo methods, but existing approaches do not yet provide the kind of comprehensive, robust, generally and automatically applicable computational

Jarad Niemi is Assistant Professor, Department of Statistics \& Applied Probability, University of California, Santa Barbara, Santa Barbara, CA 93106-3110 (E-mail: jarad@stat.duke.edu). Mike West is Arts \& Sciences Professor of Statistical Science, Department of Statistical Science, Duke University, Durham, NC 27708-0251 (E-mail: mw@stat.duke.edu).

\footnotetext{
(C) 2010 American Statistical Association, Institute of Mathematical Statistics, and Interface Foundation of North America

Journal of Computational and Graphical Statistics, Volume 19, Number 2, Pages 260-280 DOI: $10.1198 /$ jcgs.2010.08117
} 
methods needed for repeat batch analysis in routine application. The above criteria for effective statistical computation are central to our primary motivating context of dynamic cellular networks, an applied field that is beginning to grow rapidly as relevant high-resolution time series data on genetic circuitry become increasingly available in single-cell and related studies (Rosenfeld et al. 2005; Golightly and Wilkinson 2005; Wilkinson 2006; Wang et al. 2010) and synthetic bioengineering (Tan et al. 2007). With this motivation, we have built on the best available analytic and Monte Carlo tools for nonlinear dynamic models to generate a novel adaptive mixture modeling method that is embedded in an overall Markov chain Monte Carlo (MCMC) strategy for posterior computation; the resulting methodology satisfies the above criteria while also being computationally efficient, and has generated most effective MCMC analyses from the viewpoint of convergence in a range of example models we have studied.

Given a specified model and a series of observations over a fixed time interval, we are interested in summaries of the posterior distribution for the full series of corresponding state vectors as well as fixed model parameters; this is the batch analysis. Sequential filtering and retrospective smoothing using Monte Carlo is central to our general, nonlinear forward filtering, backward sampling (FFBS) approach that extends the profoundly useful FFBS methodology of conditionally linear, normal models introduced in the work of Carter and Kohn (1994) and Frühwirth-Schnatter (1994); see also West and Harrison (1997, chap. 15). Filtering is the canonical setting for sequential particulate methods (West 1992; Gordon, Salmond, and Smith 1993; West 1993b; Chen and Liu 2000; Doucet, de Freitas, and Gordon 2001; Liu and West 2001); retrospective smoothing analysis has been explored in such contexts, in terms of both marginal (Kitagawa 1996; Hürzeler and Künsch 1998; Doucet, Godsill, and Andrieu 2000) and joint (Godsill, Doucet, and West 2004) smoothing approaches. We do not, however, use particle filtering methods; though useful and interesting, the well-known shortcomings of these methods, including the key issue of particle attrition, are currently simply limiting from the viewpoint we have of robust, stable, and automatically applicable methods for a range of nonlinear models. Previous approaches using MCMC in nonlinear non-Gaussian state-space models include the works of Carlin, Polson, and Stoffer (1992), Geweke and Tanizaki (1999), and Stroud, Müller, and Polson (2003). In particular, Stroud, Müller, and Polson (2003) suggested sampling latent states in blocks from an auxiliary mixture model for use as a Metropolis proposal. The main difference of our method is that our MCMC scheme does not condition on mixture component indicators. Closer to our perspective is the work of Ravines, Migon, and Schmidt (2007) who, in the class of dynamic generalized linear models (West and Harrison 1997, chapter 14) developed a global Metropolis-Hastings analysis where the proposal distribution for state vectors is generated from analytic approximations to filtering and smoothing distributions that are known to be accurate, and hence can be expected to lead to reasonable acceptance rates. We develop this perspective using adaptive mixture approximations to filtering distributions that apply widely. This leads to accurate, direct analytic approximations to the smoothed distributions for the full set of states in a batch analysis, and hence to effective Monte Carlo that uses these approximations as proposal distributions. This sampling strategy for latent states is embedded in an overall MCMC that couples in samplers for fixed model parameters to define a complete analysis. 
Section 2 introduces the state-space model context, focusing on nonlinear models with additive Gaussian noise. Section 3 reviews mixture model approximations in state-space models, and develops a regenerating procedure to improve the utility of Gaussian sum mixtures in adaptive mixture modeling for analytic approximation to sequential filtering and smoothing. Section 4 then embeds the mixture analysis in an overall MCMC as the novel Metropolis proposal method for latent states. Section 5 illustrates the analyses with nonlinear models relevant in systems biological studies of dynamic cellular networks. Section 5.2 provides a full Bayesian analysis of a model with fixed parameters and a two-dimensional state vector in a systems biology example. Comments on, and comparisons with, prior methods are included throughout. Section 6 provides summary comments.

\section{STATE-SPACE MODEL AND FFBS ANALYSIS}

Begin with the Markovian state-space model (West and Harrison 1997)

$$
\begin{aligned}
& y_{t}=f_{t}\left(x_{t}\right)+v_{t} \quad \text { (observation equation), } \\
& x_{t}=g_{t}\left(x_{t-1}\right)+\omega_{t} \quad \text { (state evolution equation), }
\end{aligned}
$$

where $x_{t}$ is the unobserved state of the system at time $t, y_{t}$ is the observation at time $t, f_{t}(\cdot)$ and $g_{t}(\cdot)$ are known, nonlinear observation and evolution functions, and $v_{t} \sim N\left(0, V_{t}\right)$ and $\omega_{t} \sim N\left(0, W_{t}\right)$ are independent and mutually independent Gaussian terms, the observation and evolution noise terms, respectively. Initially we assume any model parameters in the nonlinear functions or noise variances are known. We note that the development can be extended well beyond our focus here on additive, normal noise terms, though for specificity we focus on that structure here.

We use $s: t$ to denote consecutive times $s, s+1, \ldots, t$ for any $s, t>s$, so that $x_{s: t}=$ $\left\{x_{s}, \ldots, x_{t}\right\}$ and so forth. Based on the batch of data $y_{1: T}$, the main goal is simulation of the set of states $x_{0: T}$ from the implied posterior

$$
p\left(x_{0: T} \mid y_{1: T}\right) \propto p\left(x_{0}\right) \prod_{t=1}^{T} p\left(y_{t} \mid x_{t}\right) p\left(x_{t} \mid x_{t-1}\right),
$$

where $p\left(x_{0}\right)$ is the density of the initial state and $p\left(y_{t} \mid x_{t}\right)$ and $p\left(x_{t} \mid x_{t-1}\right)$ are defined by equation (2.1). This is done using the FFBS strategy:

- FF: For each $t=1: T$ in sequence, sequentially process the datum $y_{t}$ to update numerical summaries of the filtering densities $p\left(x_{t} \mid y_{1: t}\right)$ at time $t$.

- $B S$ : Simulate the joint distribution in (2.2) via the implied backward compositional form

$$
p\left(x_{0: T} \mid y_{1: T}\right) \propto p\left(x_{T} \mid y_{1: T}\right) \prod_{t=1}^{T} p\left(x_{t-1} \mid x_{t}, y_{1:(t-1)}\right) .
$$

That is:

(a) draw $x_{T} \sim p\left(x_{T} \mid y_{1: T}\right)$ and set $t=T$; 
(b) draw $x_{t-1} \sim p\left(x_{t-1} \mid x_{t}, y_{1:(t-1)}\right)$;

(c) reduce $t$ to $t-1$ and return to step (b); stop when $t=0$.

This generates the full joint sample $x_{0: T}$ in reverse order. All steps of FFBS depend fundamentally on the structure of the joint densities

$$
p\left(y_{t}, x_{t}, x_{t-1} \mid y_{1:(t-1)}\right)=p\left(y_{t} \mid x_{t}\right) p\left(x_{t} \mid x_{t-1}\right) p\left(x_{t-1} \mid y_{1:(t-1)}\right) .
$$

In particular, filtering relies on the ability to compute and summarize

$$
\begin{aligned}
p\left(x_{t} \mid y_{1: t}\right) & \propto p\left(y_{t} \mid x_{t}\right) \int p\left(x_{t} \mid x_{t-1}\right) p\left(x_{t-1} \mid y_{1:(t-1)}\right) d x_{t-1} \\
& =\int p\left(y_{t}, x_{t}, x_{t-1} \mid y_{1:(t-1)}\right) d x_{t-1}
\end{aligned}
$$

while backward sampling relies on the ability to simulate from

$$
p\left(x_{t-1} \mid x_{t}, y_{1:(t-1)}\right) \propto p\left(x_{t-1} \mid y_{1:(t-1)}\right) p\left(x_{t} \mid x_{t-1}\right)=p\left(x_{t}, x_{t-1} \mid y_{1:(t-1)}\right),
$$

the bivariate margin of (2.4). In linear, Gaussian models, these distributions are all Gaussian; in nonlinear models, the implied computations require some form of approximation.

\section{NORMAL MIXTURE MODEL APPROXIMATIONS}

\subsection{BACKGROUND AND NOTATION}

Our strategy is based on approximation of the sequentially updated distributions of states via mixtures of many, very precise normal components. Mixtures have been used broadly in dynamic modeling, for both model specification and computational methods, especially in adaptive multiprocess models and to represent model uncertainty in terms of multiple models analyzed in parallel (chapter 12 and references in West and Harrison 1997; Fearnhead and Meligkotsidou 2007). The basic idea of normal mixture approximation in nonlinear state-space models in fact goes back several decades to at least the article by Harrison and Stevens (1971) in statistics and that by Sorenson and Alspach (1971) in engineering, the latter using the term Gaussian sum for direct analytic approximations to nonlinear models; see also the articles by Alspach and Sorenson (1972) and Harrison and Stevens (1976). Our method here is a direct extension of the original Gaussian sum approximation idea now embedded in the Markov chain Monte Carlo framework. The approach builds on the concept of using mixtures of many precise normal components to approximate sequences of posterior distributions for sets of states as the conditioning data are updated; in essence, this revisits and revises earlier adaptive importance sampling approaches (West 1992, 1993a, 1993b) to be based on far more efficient—computationally and statistically-Metropolis accept/reject methods.

By way of notation we denote a normal mixture distribution for a random variate $z$ by

$$
z \sim N m\left(p_{1: J}, m_{1: J}, C_{1: J}\right) \quad \text { when } p(z)=\sum_{j=1}^{J} p_{j} N\left(m_{j}, C_{j}\right),
$$


where $N\left(\mu, \sigma^{2}\right)$ indicates a Gaussian distribution with mean $\mu$ and standard deviation $\sigma$.

\subsection{Mixtures in State-Space Models}

Suppose at time $t-1$ the density $p\left(x_{t-1} \mid y_{1:(t-1)}\right)$ is—either exactly or approximatelygiven by

$$
\left(x_{t-1} \mid y_{1:(t-1)}\right) \sim N m\left(p_{t-1,1: J}, m_{t-1,1: J}, C_{t-1,1: J}\right) .
$$

Then the key trivariate density of (2.4) is

$$
p\left(y_{t}, x_{t}, x_{t-1} \mid y_{1:(t-1)}\right)=\sum_{j=1}^{J} p_{t-1, j} p\left(y_{t} \mid x_{t}\right) p\left(x_{t} \mid x_{t-1}\right) N\left(x_{t-1} \mid m_{t-1, j}, C_{t-1, j}\right) .
$$

Suppose that component variances $C_{t-1,1: J}$ are very small relative to the variances $V_{t}, W_{t}$ and inversely related to the local gradients of the regression and evolution functions $f_{t}(\cdot), g_{t}(\cdot)$ in (2.1); this generally requires a large value of $J$. Then variation of component $j$ of the summand in (3.1) is heavily restricted to the implied, small region around $x_{t-1}=m_{t-1, j}$ and we can accurately approximate $g_{t}(\cdot)$ and $f_{t}(\cdot)$ with local linearization valid in that small region. The two lead terms in summand $j$ are replaced by the local normal, linear forms $\left(x_{t} \mid x_{t-1}\right) \sim N\left(a_{t, j}+g_{t}^{\prime}\left(m_{t-1, j}\right)\left(x_{t-1}-m_{t-1, j}\right), W_{t}\right)$ with $a_{t, j}=g_{t}\left(m_{t-1, j}\right)$ and $\left(y_{t} \mid x_{t}\right) \sim N\left(f_{t, j}+f_{t}^{\prime}\left(a_{t, j}\right)\left(x_{t}-a_{t, j}\right), V_{t}\right)$ with $f_{t, j}=f_{t}\left(a_{t, j}\right)$. This immediately reduces (3.1) to a mixture of trivariate normals, so that all marginals and conditionals are computable as normal mixtures. In particular, the key distributions for filtering and smoothing are:

Equation (2.5) for forward filtering:

$$
\left(x_{t} \mid y_{1: t}\right) \sim N m\left(p_{t, 1: J}, m_{t, 1: J}, C_{t, 1: J}\right)
$$

having elements $m_{t, j}=a_{t, j}+A_{t, j}\left(y_{t}-f_{t, j}\right)$ and $C_{t, j}=R_{t, j}-A_{t, j}^{2} Q_{t, j}$ where $A_{t, j}=$ $R_{t, j} f_{t}^{\prime}\left(a_{t, j}\right) / Q_{t, j}, R_{t, j}=C_{t-1, j}\left[g_{t}^{\prime}\left(m_{t-1, j}\right)\right]^{2}+W_{t}$, and $Q_{t, j}=R_{t, j}\left[f_{t}^{\prime}\left(a_{t, j}\right)\right]^{2}+V_{t}$. The component probabilities are updated via $p_{t, j} \propto p_{t-1, j} N\left(y_{t} \mid f_{t, j}, Q_{t, j}\right)$.

Equation (2.6) for backward sampling:

$$
\left(x_{t-1} \mid x_{t}, y_{1:(t-1)}\right) \sim N m\left(q_{t, 1: J}, h_{t, 1: J}, H_{t, 1: J}\right)
$$

having elements $h_{t, j}=m_{t-1, j}+B_{t, j}\left(x_{t}-a_{t, j}\right)$ and $H_{t, j}=C_{t-1, j}-B_{t, j}^{2} R_{t, j}$ where $B_{t, j}=$ $C_{t-1, j} g_{t}^{\prime}\left(m_{t-1, j}\right) / R_{t, j}$; the component probabilities are $q_{t, j} \propto p_{t-1, j} N\left(x_{t} \mid a_{t, j}, R_{t, j}\right)$.

For large $J$ and small enough $C_{t-1, j}$, the implied filtering computations will provide good approximations to the true model analysis, and have been used quite widely in applications in engineering and elsewhere for some years. Smoothing computations based on the approximations are direct, but have been less widely used and exploited to date. Our strategy is to embed these mixture computations in an overall MCMC, using them to define a global Metropolis proposal distribution for $p\left(x_{0: T} \mid y_{1: T}\right)$. As a nice by-product, the observed Metropolis acceptance rates also provide an indirect assessment of the adequacy of the Gaussian method as a direct analytic approximation, though our interest is its use in obtaining exact posterior samples. 


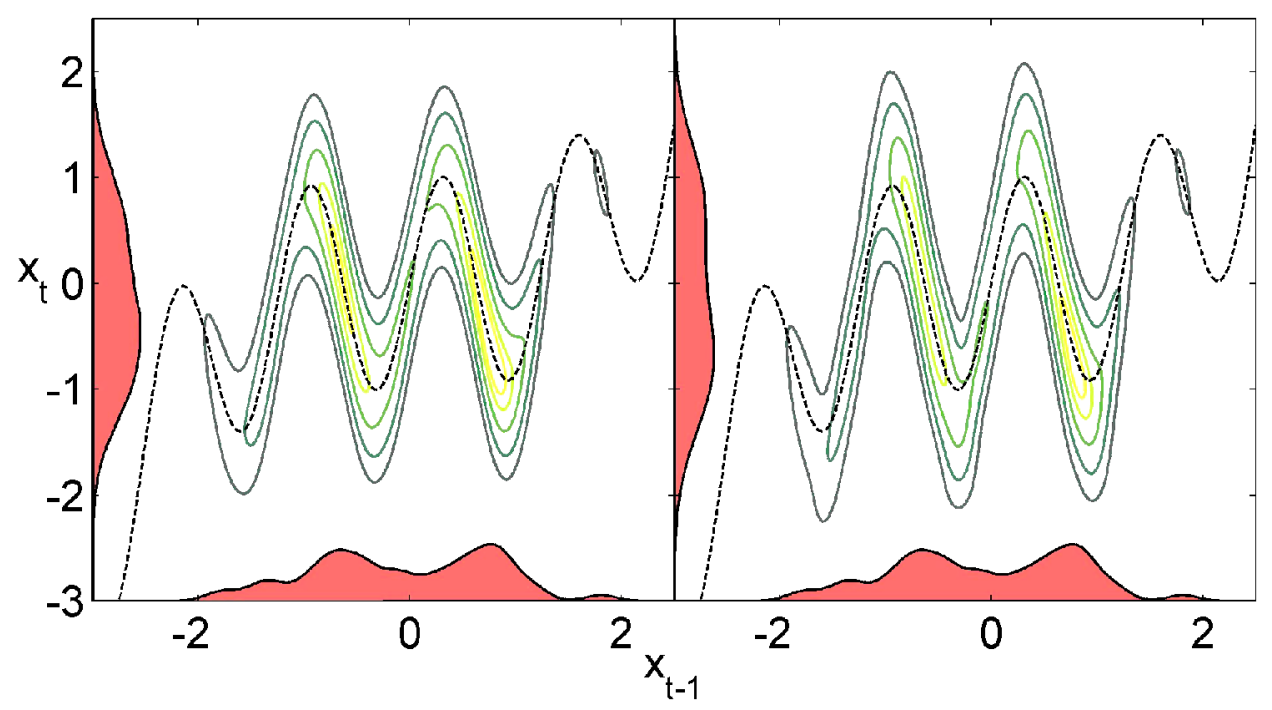

Figure 1. Bivariate and marginal distributions for two states in a model with $g_{t}(x)=0.1 x^{3}+\sin (5 x)$ and $W_{t}=0.2$, and where $p\left(x_{t-1} \mid y_{1:(t-1)}\right)$ is a $J=50$ component mixture with density graphed on the horizontal axis of each frame. The left panel shows exact bivariate density contours and, on the vertical axis, the implied margin for $\left(x_{t} \mid y_{1:(t-1)}\right)$. The right panel shows the corresponding 50-component mixture approximations. In each frame, the dashed line shows the evolution function $g_{t}(\cdot)$. A color version of this figure is available in the electronic version of this article.

\subsection{TWo-STATE EXAMPLE}

To clearly fix ideas, Figure 1 shows aspects of an example with $g_{t}(x)=0.1 x^{3}+\sin (5 x)$, $W_{t}=0.2$ and in which $\left(x_{t-1} \mid y_{1:(t-1)}\right)$ is a $J=50$ component mixture with resulting density graphed in the figure. The comparison between the bivariate contours of the exact and mixture approximation of $p\left(x_{t}, x_{t-1} \mid y_{1:(t-1)}\right)$ demonstrates the efficacy of the method in this highly nonlinear model. Evidently, the mixture model is an accurate representation of the true nonlinear model, though the joint mixture density is in fact very slightly more diffuse- a good attribute from the viewpoint of the goal of generating a useful Metropolis proposal distribution. Approximation accuracy increases with the number of normal mixture components used so long as the variances $C_{t-1, j}$ fall off appropriately as $J$ increases, as we discuss further below.

\subsection{Regenerating MiXtures}

The basic mixture approximation strategy can work well when the component means are spread out, the component variances are small, and the component probabilities are approximately equal. The component means being spread out leads to desirable wide-ranging evaluations of the nonlinear evolution and observation functions. Very small component variances improve the validity of the local linear approximations to the nonlinear functions over increasingly small regions. Balanced component probabilities ensure that each component contributes to the mixture after propagation through the nonlinearities; if only a few components dominate, all other components are effectively irrelevant and the overall strategy will collapse. 
Table 1. Regenerating procedure to approximate an arbitrary density $p(x)$ with a mixture of Gaussians $N m\left(p_{1: J}, m_{1: J}, C_{1: J}\right)$.

1. Set $p_{j}=1 / J$ for $j \in\{1, \ldots, J\}$.

2. Set $m_{j}$ equal to the $j / J+1$ quantile of $p(x)$ for $j \in\{1, \ldots, J\}$. That is,

$$
\frac{j}{J+1}=\int_{\infty}^{m_{j}} p(x) d x .
$$

3. Set $C_{j}=C$ such that

$$
\operatorname{var} X=\sum_{j=1}^{J} p_{j}\left[C+\left(m_{j}-\bar{m}\right)^{2}\right],
$$

where $\bar{m}=\sum_{j=1}^{J} p_{j} m_{j}$.

These properties are explicitly maintained using a novel regenerating procedure shown in Table 1 . Suppose we wish to approximate an arbitrary density $p(x)$ using an equally weighted mixture of Gaussians with means set at the $J+1$-quantiles of $p(x)$ and component variances constant and chosen so that the variance of the mixture equals that of $p(x)$. For large $J$, this satisfies the above desiderata for mixture approximations in our context, and this idea is used to map any given mixture distribution to one with any number of components with these characteristics.

In our model of (2.1), suppose an initial prior $p\left(x_{0}\right)$ is defined as a mixture, either directly or by applying the regeneration procedure in Table 1 to an original prior and using a large value $J$. We proceed through the sequential updating analysis, now at each step using the regenerating procedure when necessary to revise, balance, and hence improve the overall adequacy of the approximation at each stage. Depending on the model and data, this regeneration may be needed to approximate the prior $p\left(x_{t} \mid x_{t-1}, y_{1:(t-1)}\right)$ and posterior $p\left(x_{t} \mid y_{1: t}\right)$ at each step. Although (3.1) is not satisfied in these cases, (3.2) and (3.3) are still relevant and therefore a proposal can be drawn and the Metropolis acceptance probability computed.

\section{METROPOLIS MCMC}

\subsection{Adaptive MiXture Model Metropolis for States}

The mixture modeling strategy defines a computationally feasible method for evaluating and sampling from a useful approximation to the full joint posterior density of states of (2.2) and, in reverse form, (2.3). Forward filtering computations apply to sequentially update the mixture forms $p\left(x_{t} \mid y_{1: t}\right)$ over $t=1: T$ using (3.2) and the regenerating procedure. This is followed by backward sampling over $t=T, T-1, \ldots, 0$ using the mixture forms of (3.3). Write $q\left(x_{0: T} \mid y_{1: T}\right)$ for the implied joint density of states from this analysis; that is, $q\left(\cdot \mid y_{1: T}\right)$ has the form of the reverse (2.3) in which each $p(\cdot \mid \cdot)$ is replaced by the corresponding mixture density. 
We treat the analysis via Metropolis-Hastings MCMC analysis. With a current sample of states $x_{0: T}$, apply the FFBS to generate a new, candidate draw $x_{0: T}^{*}$ from the proposal distribution with density $q\left(x_{0: T} \mid y_{1: T}\right)$. This is assessed via the standard accept/reject test, accepting $x_{0: T}^{*}$ with probability

$$
\rho\left(x_{0: T}^{*} \mid x_{0: T}\right)=\min \left\{1, w\left(x_{0: T}^{*}\right) / w\left(x_{0: T}\right)\right\},
$$

where $w(\cdot)=p\left(\cdot \mid y_{1: T}\right) / q\left(\cdot \mid y_{1: T}\right), p\left(\cdot \mid y_{1: T}\right)$ is given by $(2.1)$, and $q\left(x_{0: T}^{*} \mid y_{1: T}\right)$ is given by (3.3). With no unknown fixed parameters, $q\left(x_{0: T} \mid y_{1: T}\right)$ is known from the previous MCMC iteration; otherwise the densities to evaluate $q\left(x_{0: T} \mid y_{1: T}\right)$ will need to be calculated by repeating (3.3) for $x_{0: T}$.

This is a global MCMC, applying to the full set of consecutive states, that will generally define an ergodic Markov chain on the $x_{0: T}$ based on everywhere-positivity of both $p\left(x_{0: T} \mid y_{1: T}\right)$ and $q\left(x_{0: T} \mid y_{1: T}\right)$. As $q$ is expected to provide a good global approximation, the resulting MCMC can be expected to perform well, and as mentioned above the acceptance rates provide some indication of the accuracy of the approximation. Evidently, acceptance rates can generally be expected to decay with increasing time series length $T$. Experiences of Ravines, Migon, and Schmidt (2007) in the simpler DGLM context, of our own group in this and related model contexts, bear out the utility of the method. Some additional comments and numerical comparisons of acceptance rates appear later. The overall procedure for latent state sampling is termed adaptive mixture modeling Metropolis method (AM4) and is provided in Table 2. In this algorithm, regenerate refers to the procedure in Table 1. Depending on the model and prior, these regeneration procedures may be unnecessary.

\subsection{Combined MCMC for States and Fixed Model Parameters}

Practical applications involve models with fixed, uncertain parameters as well as the latent states, and a complete analysis embeds the above simulator for states within an overall MCMC that also includes parameters (e.g., see West and Harrison 1997, sec. 15.2). With a vector of parameters $\theta$, extend the model notation to

$$
y_{t}=f_{t}\left(x_{t} \mid \theta\right)+v_{t}, \quad x_{t}=g_{t}\left(x_{t-1} \mid \theta\right)+\omega_{t},
$$

where, now, the initial prior $p\left(x_{0} \mid \theta\right)$ may involve elements of $\theta$ as may the variances $V_{t}, W_{t}$ (one key case being constant, unknown variances that are then elements of $\theta$ ).

The overall computational strategy is then to apply the above state sampler at each stage of an overall MCMC conditional on $\theta$, and to couple this with sampling of $\theta$ values using the implied distribution $p\left(\theta \mid x_{0: T}, y_{1: T}\right)$ at each step of the chain. Since $\theta$ is changing at each step in the MCMC, the filtered distributions, that is, component probabilities, means, and variances in (3.2), are recomputed at each iteration of the MCMC for joint sampling of $x_{0: T}$. Depending on the model form and priors specified for $\theta$, sampling fixed parameters will typically be performed in terms of a series of Gibbs sampling steps, perhaps with some blocking of subsets of parameters. Under a specified prior $p(\theta)$, the complete conditional posterior for any subset of elements $\theta_{i}$ given the remaining elements $\theta_{-i}$ 
Table 2. AM4 algorithm to sample from the full posterior of states given in (2.2) for the model of (2.1).

1. Regenerate $p\left(x_{0}\right)$ to obtain $p\left(x_{0}\right) \approx N m\left(p_{0,1: J}, m_{0,1: J}, C_{0,1: J}\right)$.

2. For $t=1: T$, perform the forward filtering steps:

(a) For all $j$, set

$$
\begin{aligned}
\tilde{a}_{t, j} & =g_{t}\left(m_{t-1, j}\right), \quad \tilde{R}_{t, j}=C_{t-1, j}\left[g_{t}^{\prime}\left(m_{t-1, j}\right)\right]^{2}+W_{t}, \\
\tilde{p}_{t-1, j} & =p_{t-1, j} .
\end{aligned}
$$

(b) Regenerate $\operatorname{Nm}\left(\tilde{p}_{t-1,1: J}, \tilde{a}_{t, j}, \tilde{R}_{t, j}\right)$ to obtain

$$
p\left(x_{t} \mid x_{t-1}, y_{1:(t-1)}\right) \approx N m\left(\hat{p}_{t-1,1: J}, a_{t, j}, R_{t, j}\right) .
$$

(c) For all $j$, set

$$
\begin{aligned}
f_{t, j} & =f_{t}\left(a_{t, j}\right), \quad Q_{t, j}=R_{t, j}\left[f_{t}^{\prime}\left(a_{t, j}\right)\right]^{2}+V_{t}, \\
\tilde{m}_{t, j} & =a_{t, j}+A_{t, j}\left(y_{t}-f_{t, j}\right), \quad \tilde{C}_{t, j}=R_{t, j}-A_{t, j}^{2} Q_{t, j}, \\
\tilde{p}_{t, j} & \propto \hat{p}_{t-1, j} N\left(y_{t} \mid f_{t, j}, Q_{t, j}\right),
\end{aligned}
$$

where $A_{t, j}=R_{t, j} f_{t}^{\prime}\left(a_{t, j}\right) / Q_{t, j}$.

(d) Regenerate $\operatorname{Nm}\left(\tilde{p}_{t, 1: J}, \tilde{m}_{t, j}, \tilde{C}_{t, j}\right)$ to obtain

$$
p\left(x_{t} \mid y_{1: t}\right) \approx N m\left(p_{t, 1: J}, m_{t, j}, C_{t, j}\right) .
$$

3. Draw $x_{T}^{*} \sim N m\left(p_{T, 1: J}, m_{T, 1: J}, C_{T, 1: J}\right)$.

4. For $t=(T-1): 0$, perform the backward sampling draws:

(a) For all $j$, set

$$
\begin{aligned}
h_{t, j} & =m_{t-1, j}+B_{t, j}\left(x_{t}-\tilde{a}_{t, j}\right), \quad H_{t, j}=C_{t-1, j}-B_{t, j}^{2} \tilde{R}_{t, j}, \\
q_{t, j} & \propto \tilde{p}_{t-1, j} N\left(x_{t} \mid \tilde{a}_{t, j}, \tilde{R}_{t, j}\right),
\end{aligned}
$$

where $B_{t, j}=C_{t-1, j} g_{t}^{\prime}\left(m_{t-1, j}\right) / R_{t, j}$.

(b) Draw $x_{t}^{*} \sim N m\left(q_{t, 1: J}, h_{t, 1: J}, H_{t, 1: J}\right)$.

5. Accept $x_{0: T}^{*}$ with probability $\rho\left(x_{0: T}^{*} \mid x_{0: T}\right)$ given in (4.1).

is

$$
p\left(\theta_{i} \mid \theta_{-i}, x_{0: T}, y_{1: T}\right) \propto p(\theta) p\left(x_{0} \mid \theta\right) \prod_{t=1}^{T} p\left(y_{t} \mid x_{t}, \theta\right) p\left(x_{t} \mid x_{t-1}, \theta\right) .
$$

Sometimes this conditional can be sampled directly; a key example is when $V=V_{t}, W=$ $W_{t}$, and $\theta_{i}=(V, W)$ when, under independent inverse gamma priors, the above conditional posterior is also the product of independent inverse gammas. In other cases, resampling some elements $\theta_{i}$ will use random-walk Metropolis-Hastings methods involving an accept/reject test to resample $\theta_{i}$, that is, a standard Metropolis-within-Gibbs series of moves. So long as the prior density $p(\theta)$ can be directly and easily evaluated up to a constant, such moves are easy to implement since the terms in equation (4.2) can be trivially evaluated at any point $\theta$. Our example in Section 5.2 illustrates this overall strategy. 


\section{EXAMPLES}

\subsection{ILLUMinAting EXAMPLE}

Consider the example model with

$$
\begin{aligned}
& y_{t}=x_{t}^{2} / 20+v_{t}, \\
& x_{t}=x_{t-1} / 2+25 x_{t-1} /\left(1+x_{t-1}^{2}\right)+8 \cos (1.2 t)+\omega_{t},
\end{aligned}
$$

where $V_{t}=V=10, W_{t}=W=1$, and, initially, $x_{0} \sim N(0,10)$. This model was originally introduced by Andrade Netto, Gimeno, and Mendes (1978) and has since been studied by Kitagawa (1987), West (1993b), Gordon, Salmond, and Smith (1993), Hürzeler and Künsch (1998), Doucet, Godsill, and Andrieu (2000). Interest in this model has stemmed from the nonlinear nature found in both the observation and evolution equations. In addition, the squared term in the observation equation introduces a bimodal likelihood for $x_{t}$ whenever $y_{t}>0$. As will be seen, this causes multimodality in the resulting smoothed distribution for the states.

Simulated states and observations with $T=100$ appear in Figure 2. Using $J=1000$ and regenerating $p\left(x_{t} \mid x_{t-1}, y_{1:(t-1)}\right)$ and $p\left(x_{t} \mid y_{1: t}\right)$ at each $t$, the resulting filtering distributions are shown for selected time points in Figure 3. This figure shows filtering densities that display markedly non-Gaussian behavior, the bimodality being induced by the lack of identification of the sign of $x_{t}$ from the data alone.

AM4 applied to the 101-dimensional set of states $x_{0: T}$ generated 100,000 samples following 100,000 burn-in steps, achieving acceptance rates of around 20\%. Figure 4 shows histograms of the sampled states at the same time points in Figure 3, again evidence of the high degree of non-Gaussianity.

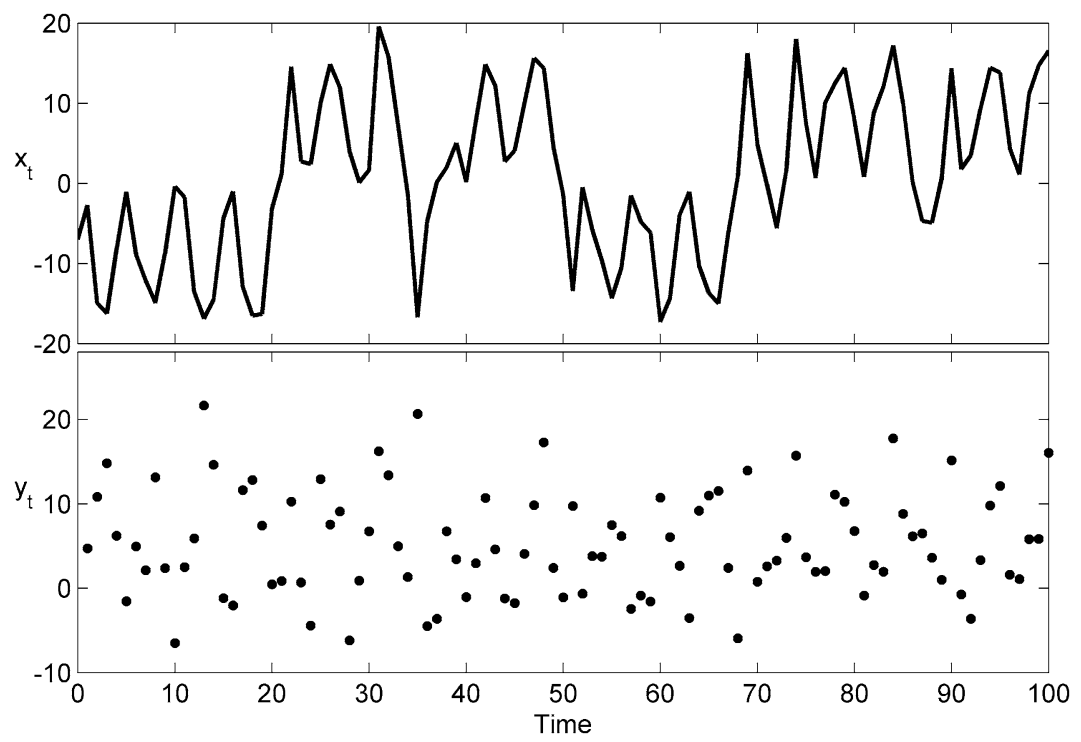

Figure 2. Model of (5.1): Simulated latent states and observations. 


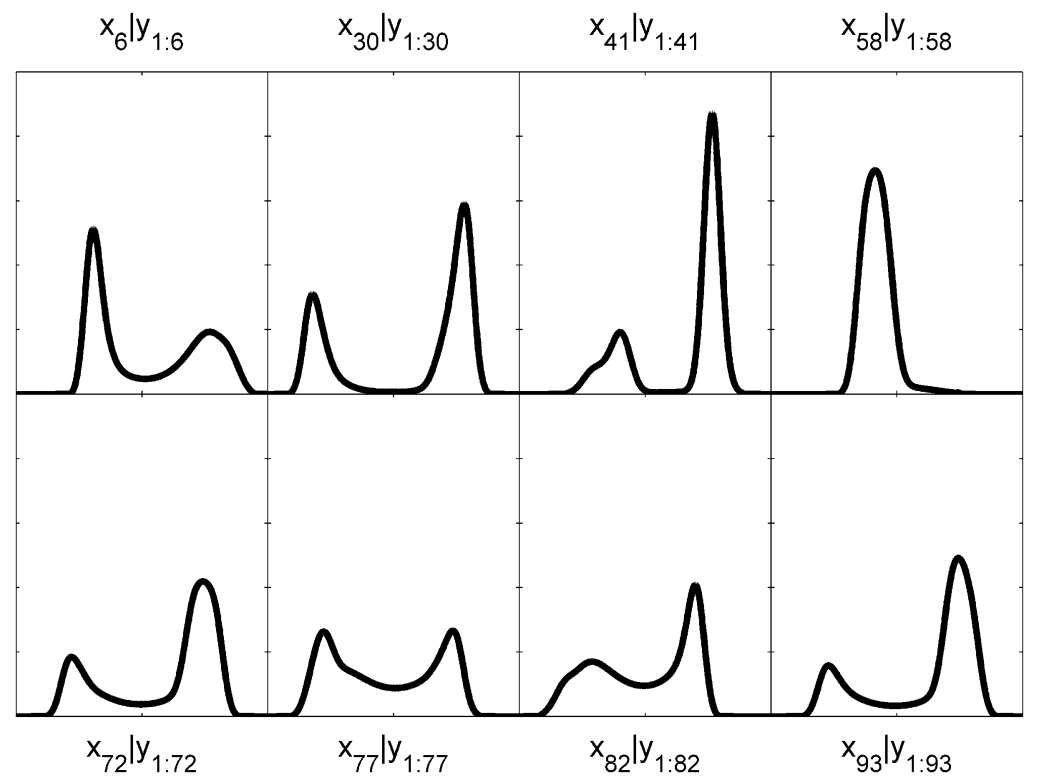

Figure 3. Model of (5.1): Filtering densities for selected time points that display markedly non-Gaussian behavior.

Figure 5 compares AM4 to the standard MCMC method in the article by Carlin, Polson, and Stoffer (1992), where univariate states are sampled, and in that by Kitagawa (1987), which evaluates the smoothed density on a grid. For state $x_{58}$ all three methods achieve equivalent results, but for state $x_{72}$ the Carlin, Polson, and Stoffer method never visits the

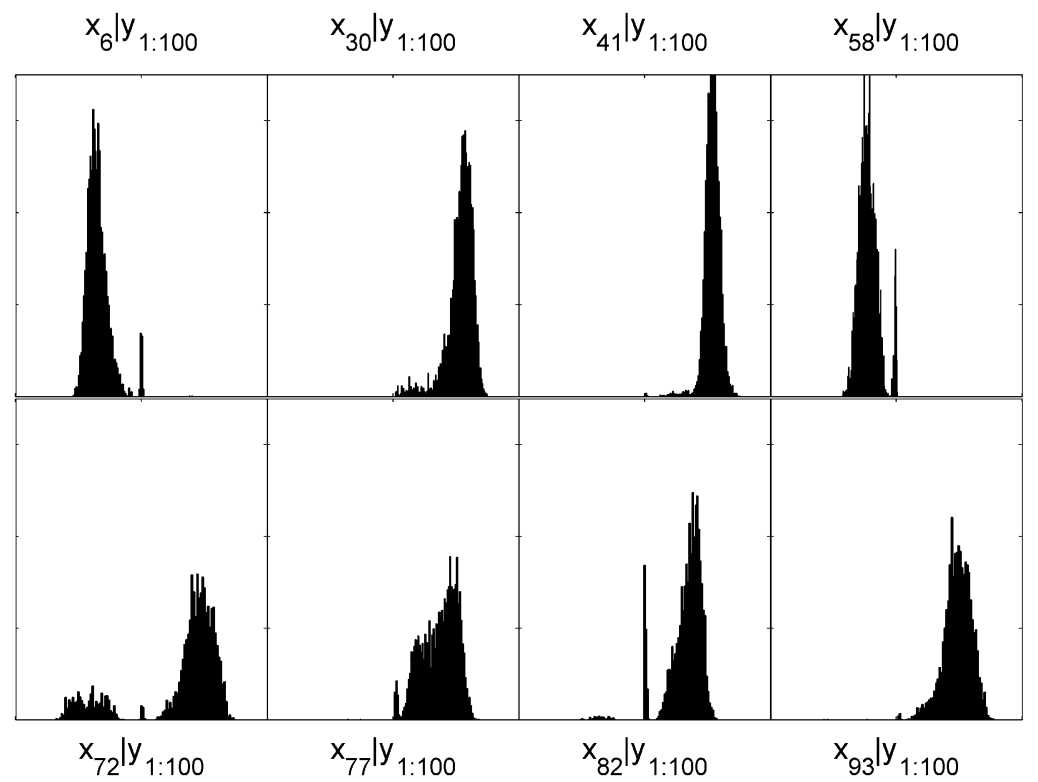

Figure 4. Model of (5.1): Histograms of the smoothed samples for the same time points shown in Figure 3. 

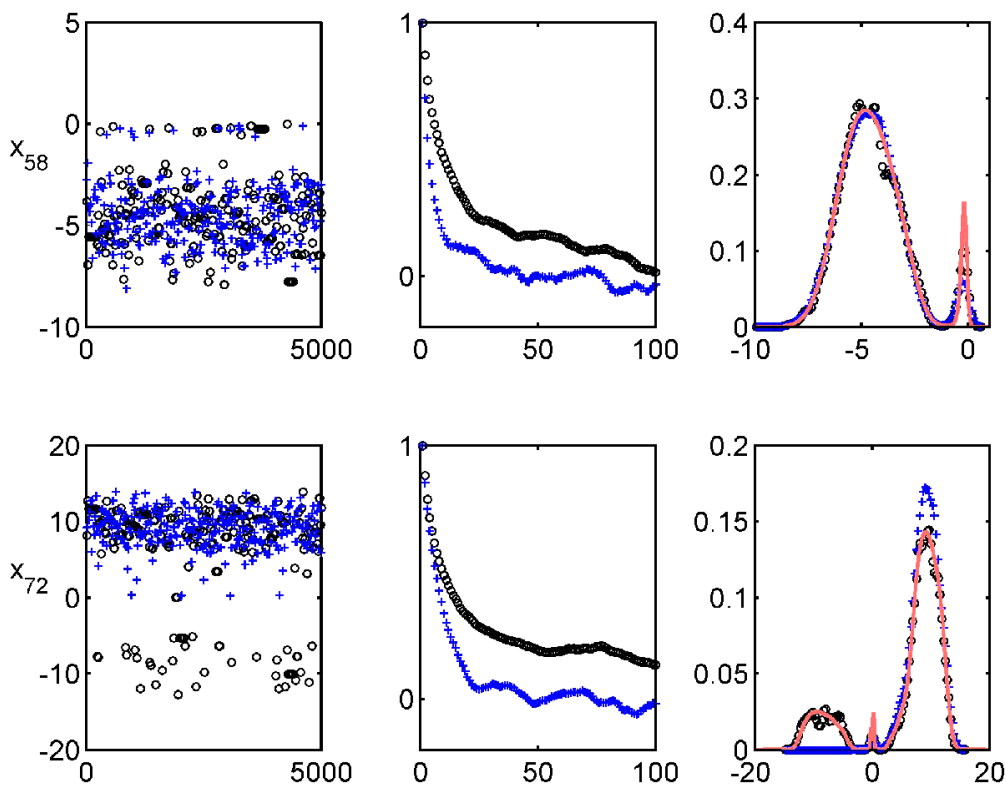

Figure 5. Model of (5.1): Traceplots, autocorrelation functions, and histograms for two different states comparing AM4 ('o'), Carlin, Polson, and Stoffer (1992) ('+'), and Kitagawa (1987) ('-'). A color version of this figure is available in the electronic version of this article.

leftmost mode of the distribution. Therefore the autocorrelations for the Carlin, Polson, and Stoffer method are misleadingly low.

More interesting are the bivariate smoothing distributions for $\left(x_{t}, x_{t-1} \mid y_{1: T}\right)$ for each $t$, obtained simply from marginal samples from the MCMC. Figure 6 shows a scatterplot of the posterior samples for $x_{71}$ versus $x_{72}$, while Figure 7 shows a smoothed density estimate for these same data.

These two figures display a high degree of non-Gaussian behavior and the reconstructions are simply not obtainable under standard linearization methods or easily, if at all, via other numerical approaches. Standard MCMC methods such as sampling univariate states or a multivariate random walk have difficulty escaping the modes shown in Figure 7. Particle filtering approaches such as that of Godsill, Doucet, and West (2004) can produce results with similar multimodal posteriors. But, for models with unknown fixed parameters such as those to follow, these methods quickly suffer from the added dimensionality.

\subsection{Example Motivated by Pathway Studies in Systems Biology}

Discrete-time models have been gaining popularity for modeling biochemical pathways. Initial uses were aimed at approximate parameter inference of stochastic differential equations (Golightly and Wilkinson 2005, 2006a, 2006b; Wilkinson 2006). More recently discrete-time models have been suggested as alternatives to ordinary and stochastic differential equations (Gadkar, Gunawan, and Doyle 2005; Gadkar, Varner, and Doyle 2005; He, Yeung, and Brown 2007, 2008). Here we analyze a discrete-time model of a biological system. Derivation of this model can be found in the work of Niemi (2009). 


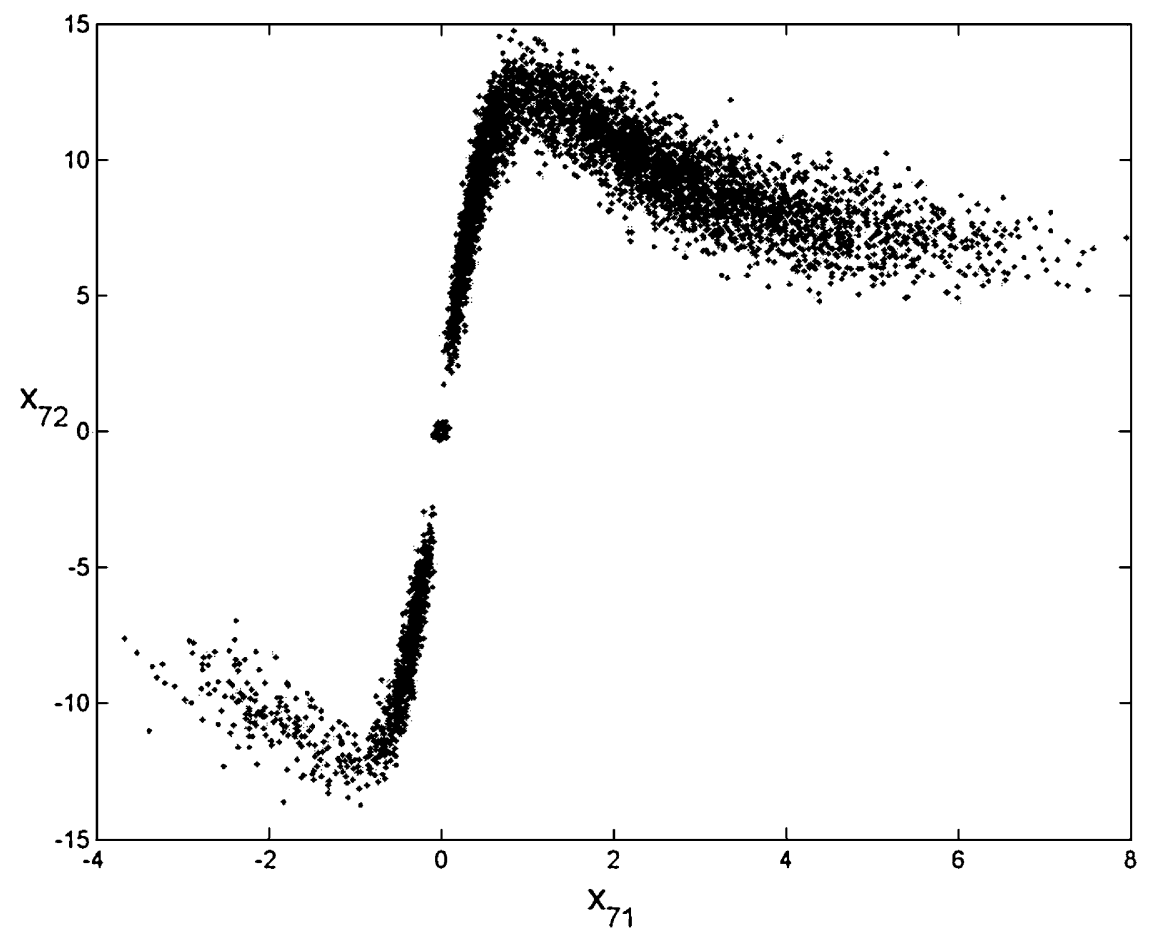

Figure 6. Model of (5.1): Scatterplot of MCMC samples for $x_{71: 72 .}$

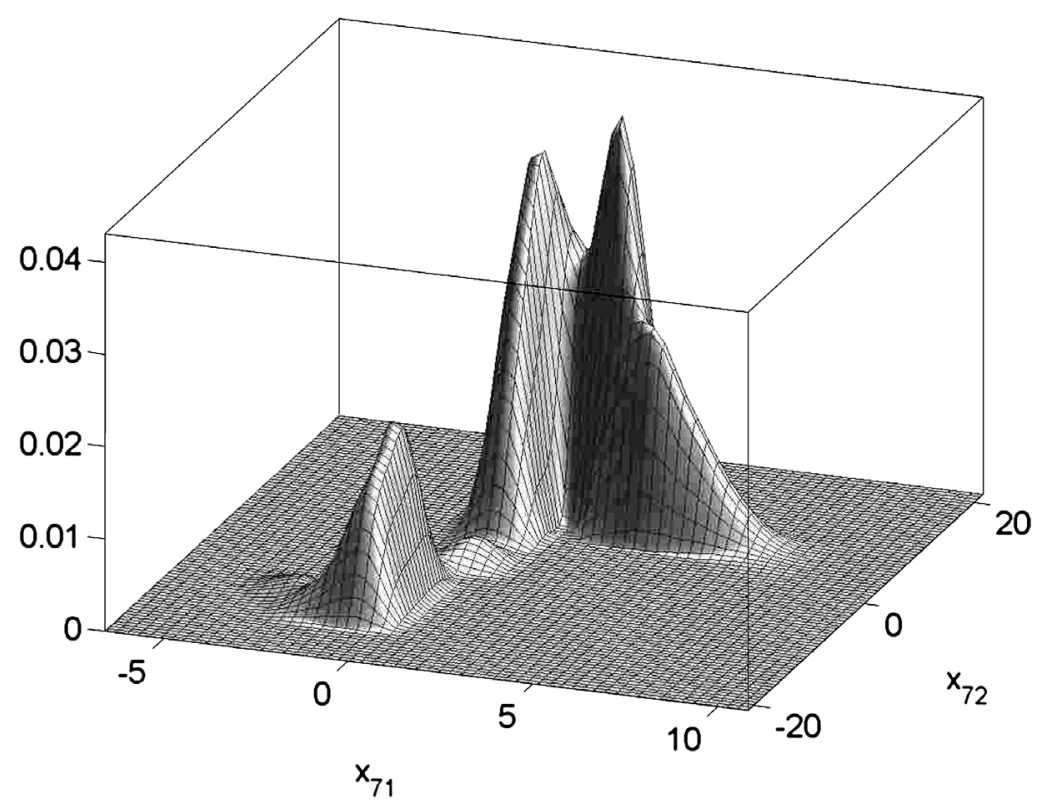

Figure 7. Model of (5.1): Reconstruction of bivariate density $p\left(x_{71,72} \mid y_{1: 100}\right)$. 
Consider a biological system that has two proteins of interest: an activator and a target. Two molecules of the activator and two molecules of the target can combine to form a tetramer. This tetramer can then enhance the production of the target. This type of system is very common in human cells, and particularly in gene pathways that control cell developmental processes that play key roles in cancer when deregulated (Sears, Ohtani, and Nevins 1997; Nevins 1998; Bild et al. 2006). Activators themselves often have an oscillating pattern-related to progression through cell cycles. As a synthetic example that mirrors this structure, suppose that we obtain noisy measurements of the activator and target proteins

$$
\begin{aligned}
y_{t} & =\left(\begin{array}{c}
a_{t} \\
x_{t}
\end{array}\right)+v_{t}, \\
\left(\begin{array}{c}
a_{t} \\
x_{t}
\end{array}\right) & =\left(\begin{array}{c}
\mu_{i_{t}}+\phi\left(a_{t-1}-\mu_{i_{t}}\right) \\
\left(k+\alpha a_{t-1}^{2} x_{t-1}^{2}\right) /\left(\beta+a_{t-1}^{2} x_{t-1}^{2}\right)+\psi x_{t-1}
\end{array}\right)+\omega_{t},
\end{aligned}
$$

where $i_{t} \in\{$ on, off $\}, v_{t} \sim N\left(0, \sigma_{m}^{2} I\right), \omega_{t} \sim N\left(0, \operatorname{diag}\left(\sigma_{a}^{2}, \sigma_{x}^{2}\right)\right)$ again independent and mutually independent, and $p\left(x_{0} \mid D_{0}\right)=N(1.5,0.5)$; here $a_{t}$ and $x_{t}$ indicate mean fluorescence levels of the activator and target proteins, respectively, at time $t$. The tetramer binding of these proteins is represented through the $a^{2} x^{2}$ term since the exponents are determined from the number of molecules of each component. To recreate the oscillating pattern seen in activators, experimental conditions are controlled such that the activator can be modeled as an autoregressive process with two distinct means, $\mu_{o n}$ and $\mu_{\text {off }}$, where the state $i_{t}$ is known at all time points. Figure 8 provides a pictorial representation of the experimental setup and synthetic gene circuit.

Typically, the evolution equation is addressed separately through their production and decay functions. Consider first the evolution equation for the target protein. $\psi x$ indicates that from one time point to the next $100(1-\psi) \%$ of the protein decays on average; decay is linear in $x$ and independent of the activator. On the other hand, the production function,
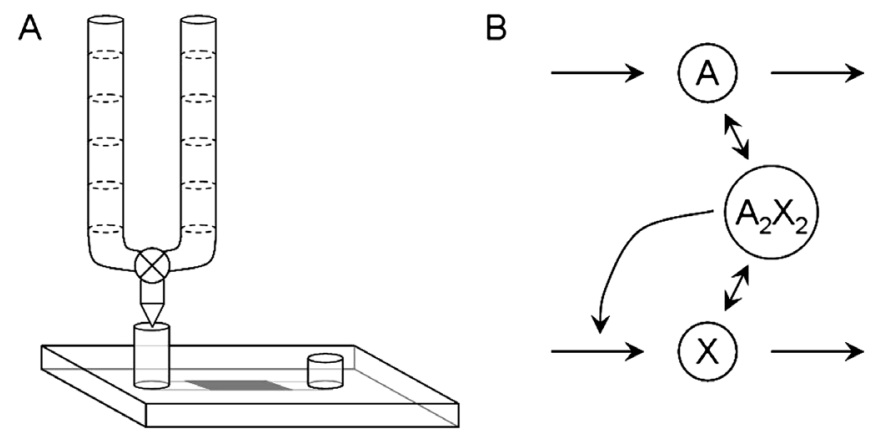

Figure 8. Model of (5.2): Depiction of a tetramer experiment. (A) Experimental setup with switched glass burets containing chemical solutions to control the on-off state of the activator. The desired solution flows into a channeled microscopy slide. Bacteria containing plasmids with a synthetic gene circuit are adhered to the gray area of the channel. (B) A synthetic gene circuit containing three chemical species: an activator, a target, and a tetramer, $A_{2} X_{2}$. Arrows indicate that the activator and target are produced, degraded, and can form tetramers. Tetramers can decay back to the activator and target or can enhance the production of the target. 


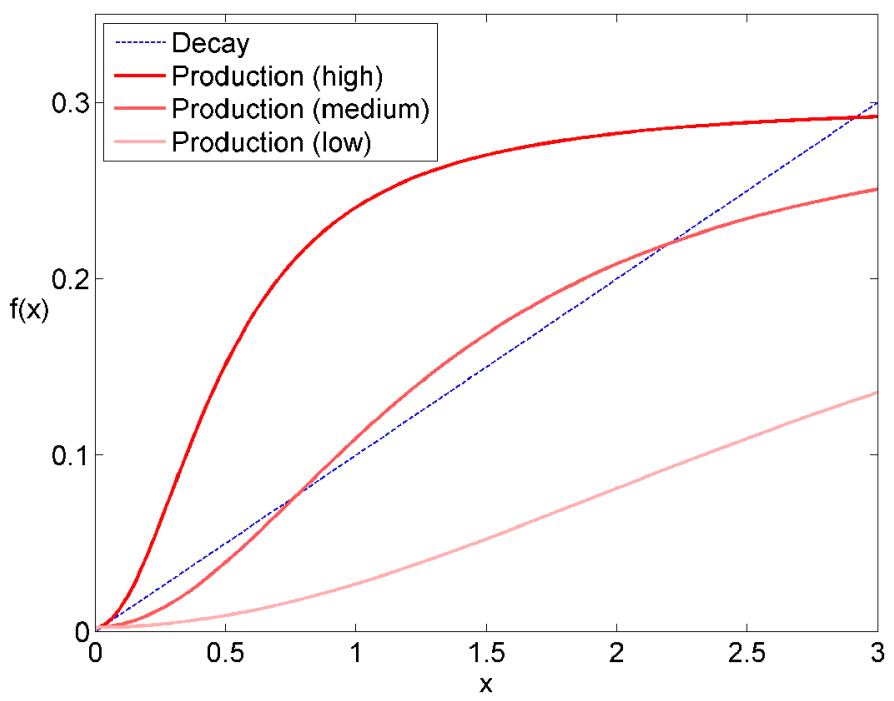

Figure 9. Model of (5.2): Production and decay functions for various levels of the activator. A color version of this figure is available in the electronic version of this article.

$\left(k+\alpha a_{t}^{2} x_{t}^{2}\right) /\left(\beta+a_{t}^{2} x_{t}^{2}\right)$, has a logistic form in $x_{t}$ and also varies depending on the activator. This logistic form implies a minimal level of target protein production to be $k / \beta$ and a maximal amount to be $\alpha$. Figure 9 shows examples for high, medium, and low levels of the activator. The figure is easiest to interpret by choosing an $x$ and then looking at whether the production line is above or below the decay line. The increase or decrease in $x$ will be, on average, proportional to the difference between these two lines. The autoregressive nature of the activator's evolution equation is a simple reparameterization of constitutive production, $\mu_{i}(1-\phi)$, and decay, $(1-\phi)$, linear in $a$. This parameterization has the interpretation that $\mu_{i}$ is the steady-state mean of the activator under the on and off experimental conditions which can be accurately estimated in steady-state experiments.

Of particular interest in these systems is the true level of the target since it may affect genes downstream in the overall biological pathway. In order to provide accurate estimates of the target protein, we need to account for uncertainty present in fixed parameters as well as the activator level. The analysis performed through MCMC is decomposed into Gibbs and Metropolis steps. The steps are all univariate with the exception of the draws for the latent states of the activator $a_{0: T}$ and the target $x_{0: T}$. With appropriate priors, most fixed parameters are available as Gibbs steps. The full conditional for $\beta$ is unavailable and hence we use a random-walk Metropolis. The joint draw for $a_{0: T}$ is available through the standard FFBS augmented with a Metropolis-Hastings step to account for the target's evolution equation. The diagonal evolution error structure allows for sampling $x_{0: T}$ through AM4.

Informative priors are used for all fixed parameters either to truncate the parameters to reasonable regions or to provide information on plausible biological knowledge. For example, parameters in the evolution of the target protein are products of chemical kinetic reaction parameters. These parameters are all positive and therefore their products are also positive. Truly informative priors are provided for some parameters including steady-state 

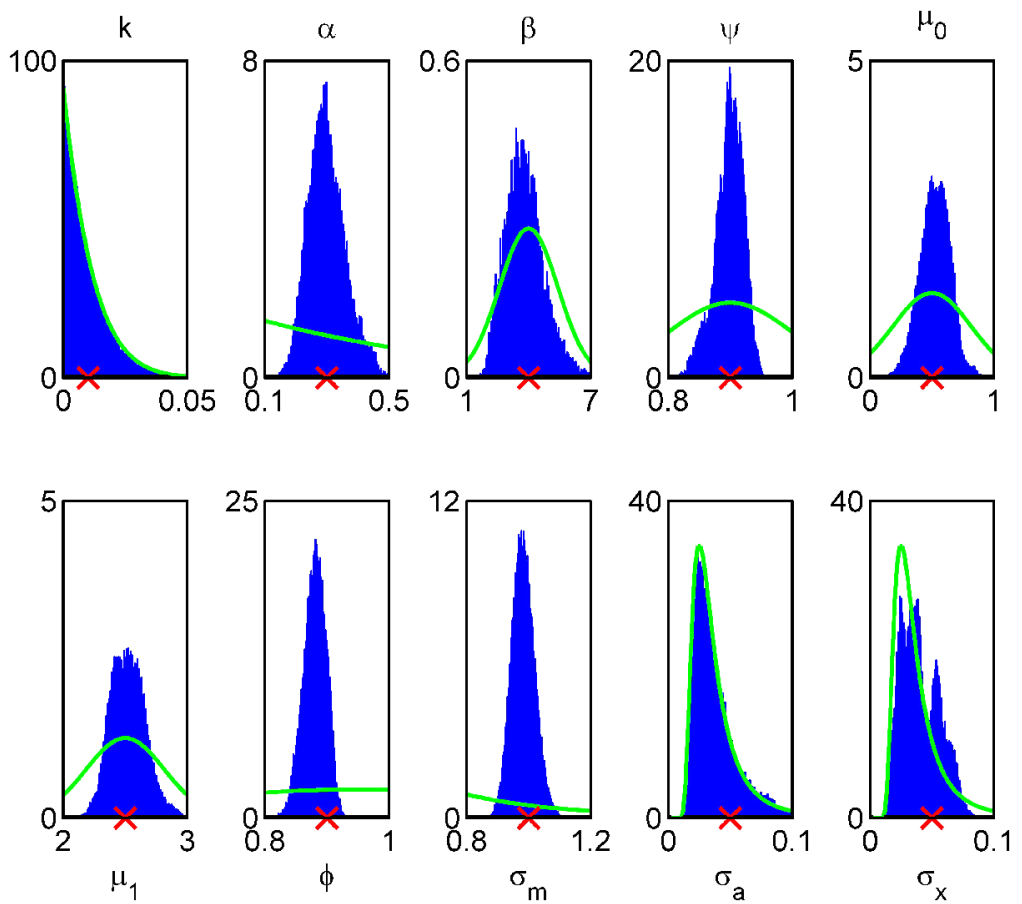

Figure 10. Model of (5.2): Histograms of marginal posterior estimates (shaded histogram), prior (superimposed curve), and true value (' $x$ ') for fixed model parameters. A color version of this figure is available in the electronic version of this article.

means of the activator in the on and off states, which could be measured with accuracy in steady-state experiments.

The full MCMC analysis was performed using $J=10$ mixture components and regeneration of both the prior and posterior at each time step and saving 50,000 iterations after 5000 burn-in. The Metropolis steps achieved acceptance probabilities of $34 \%, 46 \%$, and $8 \%$ for $\beta, x_{0: T}$, and $a_{0: T}$, respectively. Figure 10 provides posterior marginal histograms for fixed model parameters as well as their priors and true values. Figure 11 provides posterior median and pointwise $95 \%$ credible intervals for the activator and target protein. In more realistic scenarios, many of the proteins of interest may have no observations. In these situations, the methodology will work, but generally more information will need to be provided through the priors for meaningful inferences.

\section{FURTHER DISCUSSION}

The adaptive mixture modeling Metropolis method developed and exemplified here represents an efficient, effective, and relatively easily implemented computational strategy for Bayesian inference in nonlinear state-space models. For implementation, the method as presented requires only availability of first-order derivatives of the evolution and observation equation similar to the extended Kalman filter, coupled with simulation routines. Extensions to non-Gaussian models require the first two derivatives of the log-likelihood. 

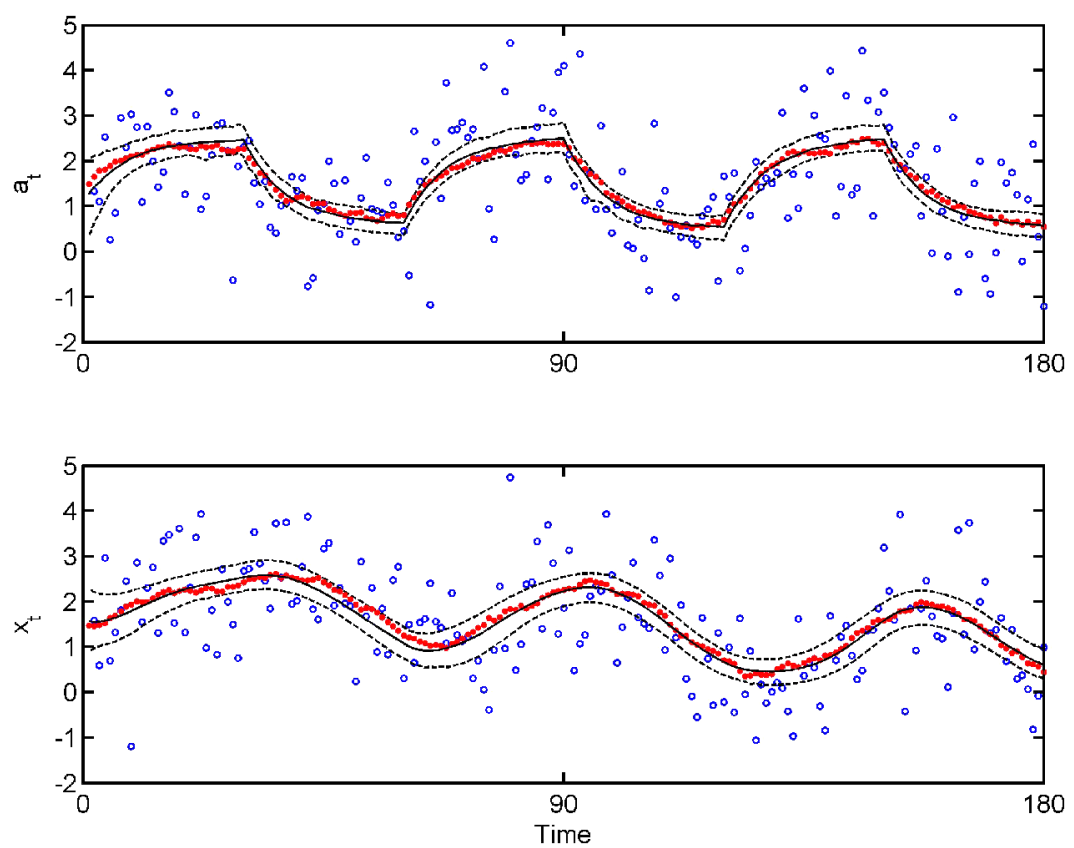

Figure 11. Model of (5.2): Pointwise median (solid line) and 95\% credible interval (dashed line) results for the underlying state (dots) and the observed data (circles). The activator is shown in the top panel and the target protein in the bottom. A color version of this figure is available in the electronic version of this article.

The overall approach represents a nice update on the use of Gaussian mixtures as direct analytic approximations and in a real sense a completion of a line of computational development for state-space models that stretches back nearly forty years. Many old ideas are good ideas, and the simple strategy of "Metropolizing" a global, analytic approximation to the full posterior distribution of a set of states adds a modern computational touch to a good, older idea. Critically, however, the mixture regeneration concept and strategy introduced here are simply fundamental to practical utility, as otherwise mixture approximations can and often will degenerate as discussed. The broader utility of the overall approach is clear once the state sampler is embedded in a larger MCMC that couples in fixed parameter sampling, as illustrated in our examples.

We have compared this method to other standard methods for analysis of state-space models including univariate state sampling and a multivariate random walk. In the example of Section 5.1, the Carlin, Polson, and Stoffer method failed to sufficiently explore the posterior over the set of states due to getting caught in modes, and similar results are obtained with random-walk proposals. Even after adjusting for computational time, AM4 outperformed these standard methods. AM4 is a widely applicable and effective methodology for MCMC analysis of state-space models.

It is worth noting that there are various other uses of adaptive mixture approximations in time series and state-space models (West and Harrison 1997). One related line of development to note is that of Chen and Liu (2000), who used mixtures of Gaussians to approximate the filtering densities for each $x_{t}$. They then proceeded with a particulate, sequential Monte Carlo approach: conditioning on state particles to induce a linearized sys- 
tem and adjusting the weights to create the filtered densities for $x_{t+1}$. That approach adds particle filtering uncertainties to the problem. The approach is closely allied to the earlier sequential adaptive importance sampling approach of West (1992, 1993a, 1993b), though replaces the uses of iteratively refined mixture approximations to posteriors for states at each time point with particle filtering. Though based on mixtures, the computational focus and method of Chen and Liu (2000) are clearly very different from our work; again, we generate an accurate, global approximation to the full set of posteriors over states using deterministic mixture approximations, and use it as a global proposal for a Metropolis step within an MCMC framework. This accomplishes fully Bayesian inference for filtering and smoothing analyses in an overarching framework that includes fixed parameters as well as dynamic states.

On matters of statistical and computational efficiency, we are encouraged by the high acceptance rates in the examples here, and a range of other studies with similar models. A nice by-product of the Metropolis strategy is that the acceptance rates can, in any specific application, be viewed as benchmarks on the inherent accuracy of the underlying analytic mixture-based posterior approximation. The trade-offs, in terms of statistical versus computational efficiencies, relate to the number of mixture components chosen and the length of the observed time series, among other things. As noted, filtering approximations with mixtures can be made arbitrarily accurate, theoretically, by increasing the number of mixture components, at the cost of cpu time increasing linearly with the number of components. Also, as is true much more widely, Metropolis acceptance rates will decrease roughly linearly on a log scale with the time series sample size; while this generic issue can be addressed with longer MCMC run lengths and subsampling-a standard response to decreased acceptance rates-modifying the underlying proposal distribution by simply increasing the number of mixture components helps also. Tables 3 and 4 provide some insights into this, with a set of empirical estimates of acceptance rates and cpu times in a simple simulated model context. Extensions and novel strategies are clearly needed to extend analyses to much longer time series while maintaining acceptance rates at practically useful levels, and some of our current research is exploring some new directions for this.

Table 3. Empirical mean (sd) $\%$ acceptance rates from 100 simulations of the model defined by: $f_{t}(x)=x$, $g_{t}(x)=\sin (x), V_{t}=1, W_{t}=1$, and $x_{0} \sim N(0,10)$.

\begin{tabular}{rrrrr}
\hline \hline & \multicolumn{4}{c}{ Length of time series } \\
\cline { 2 - 5 } Number of components & 10 & 50 & 100 & 500 \\
\hline 1 & $0(4)$ & $15(5)$ & $5(2)$ & $0(1)$ \\
5 & $62(8)$ & $42(5)$ & $27(6)$ & $2(1)$ \\
10 & $66(7)$ & $44(5)$ & $28(5)$ & $2(2)$ \\
50 & $74(6)$ & $46(10)$ & $28(11)$ & $1(2)$ \\
100 & $79(12)$ & $54(13)$ & $34(14)$ & $2(3)$ \\
\hline
\end{tabular}


Table 4. Relative empirical mean (sd) computation time in minutes for 10,000 iterations using the model in Table 3. Analysis was performed using Matlab R2007b on a $3.4 \mathrm{GHz}$ Intel Pentium 4 processor.

\begin{tabular}{rcrrr}
\hline \hline & \multicolumn{4}{c}{ Length of time series } \\
\cline { 2 - 5 } Number of components & 10 & 50 & 100 & 500 \\
\hline 1 & $0.4(0.0)$ & $1.7(0.2)$ & $3.3(0.3)$ & $15.9(1.6)$ \\
5 & $0.6(0.1)$ & $2.5(0.3)$ & $4.9(0.5)$ & $24.2(2.5)$ \\
10 & $0.6(0.1)$ & $2.7(0.3)$ & $5.2(0.6)$ & $25.7(2.7)$ \\
50 & $0.8(0.1)$ & $3.7(0.5)$ & $7.2(0.9)$ & $36.2(4.5)$ \\
100 & $1.1(0.2)$ & $5.2(0.8)$ & $10.2(1.5)$ & $51.1(7.1)$ \\
\hline
\end{tabular}

\subsection{CODE}

The web page http://ftp.stat.duke.edu/WorkingPapers/08-21.html provides freely available Matlab code that implements the method described here. This includes support functions and the examples from this article as templates for other more general models. (The version of this code used to produce this article is available on the JCGS webpage; see Supplemental Materials section for details.)

\section{SUPPLEMENTAL MATERIALS}

Nonlinear non-Gaussian models: This file describes an extension of the AM4 framework for nonlinear non-Gaussian dynamic models. It contains an example based on a stochastic volatility model. (SV.pdf)

MATLAB code for AM4: Folders contain all code and datasets necessary for executing the examples in this article. A README.txt file contains all details about the code and datasets in those folders. (code.zip)

\section{ACKNOWLEDGMENTS}

The authors thank the editor and two anonymous reviewers for their constructive suggestions that helped improve this work. We are grateful to Lingchong You and Chee-Meng Tan for discussion of dynamic models in systems biology. We acknowledge support of the National Science Foundation (grants DMS-0342172 and BES0625213) and the National Institutes of Health (grants P50-GM081883-01 and NCI U54-CA-112952-01). Any opinions, findings, and conclusions or recommendations expressed in this work are those of the authors and do not necessarily reflect the views of the NSF or NIH.

[Received August 2008. Revised March 2010.]

\section{REFERENCES}

Alspach, D. L., and Sorenson, H. W. (1972), "Non-Linear Bayesian Estimation Using Gaussian Sum Approximations," IEEE Transactions on Automatic Control, AC-17, 439-448. [263]

Andrade Netto, M. L., Gimeno, L., and Mendes, M. J. (1978), “A New Spline Algorithm for Non-Linear Filtering of Discrete Time Systems," in Proceedings of the 4th IFAC Symposium on Identification and System Parameter Estimation, Tbilisi, U.S.S.R., pp. 2123-2130. [269] 
Bild, A. H., Yao, G., Chang, J. T., Wang, Q., Potti, A., Chasse, D., Joshi, M., Harpole, D., Lancaster, J. M., Berchuck, A., Olson, J. A., Marks, J. R., Dressman, H. K., West, M., and Nevins, J. (2006), “Oncogenic Pathway Signatures in Human Cancers as a Guide to Targeted Therapies," Nature, 439, 353-357. [273]

Carlin, B. P., Polson, N. G., and Stoffer, D. S. (1992), "A Monte Carlo Approach to Nonnormal and Nonlinear State-Space Modeling,” Journal of the American Statistical Association, 87, 493-500. [261,270,271,276]

Carter, C. K., and Kohn, R. (1994), “On Gibbs Sampling for State-Space Models,” Biometrika, 81, 541-553. [261]

Chen, R., and Liu, J. S. (2000), "Mixture Kalman Filters," Journal of the Royal Statistical Society, Ser. B, 62, 493-508. [261,276,277]

Doucet, A., de Freitas, N., and Gordon, N. (eds.) (2001), Sequential Monte Carlo Methods in Practice, New York: Springer-Verlag. [261]

Doucet, A., Godsill, S., and Andrieu, C. (2000), "On Sequential Monte Carlo Sampling Methods for Bayesian Filtering," Statistics and Computing, 10, 197-208. [261,269]

Fearnhead, P., and Meligkotsidou, L. (2007), "Filtering Methods for Mixture Models," Journal of Computational and Graphical Statistics, 16, 586-607. [263]

Frühwirth-Schnatter, S. (1994), "Data Augmentation and Dynamic Linear Models," Journal of Time Series Analysis, 15, 183-202. [261]

Gadkar, K. G., Gunawan, R., and Doyle, F. J. (2005), "Iterative Approach to Model Identification of Biological Networks," BMC Bioinformatics, 6, 155. [271]

Gadkar, K. G., Varner, J., and Doyle, F. J. (2005), "Model Identification of Signal Transduction Networks From Data Using a State Regulator Problem,” IEE Systems Biology, 2, 17-30. [271]

Geweke, J., and Tanizaki, H. (1999), "On Markov Chain Monte Carlo Methods for Nonlinear and Non-Gaussian State-Space Models," Communications in Statistics: Simulation and Computation, 28, 867-894. [261]

Godsill, S. J., Doucet, A., and West, M. (2004), “Monte Carlo Smoothing for Nonlinear Time Series,” Journal of the American Statistical Association, 99, 156-168. [261,271]

Golightly, A., and Wilkinson, D. J. (2005), "Bayesian Inference for Stochastic Kinetic Models Using a Diffusion Approximation," Biometrics, 61, 781-788. [261,271]

_ (2006a), "Bayesian Sequential Inference for Nonlinear Multivariate Diffusions," Statistics and Computing, 16, 323-338. [271]

(2006b), "Bayesian Sequential Inference for Stochastic Kinetic Biochemical Network Models," Journal of Computational Biology, 13, 838-851. [271]

Gordon, N. J., Salmond, D. J., and Smith, A. F. M. (1993), "Novel Approach to Non-Linear/Non-Gaussian Bayesian State Estimation," IEE Proceedings Part F: Communications, Radar and Signal Processing, 140, 107-113. [261,269]

Harrison, P., and Stevens, C. (1971), “A Bayesian Approach to Short-Term Forecasting," Operations Research Quarterly, 22, 341-362. [263]

_ (1976), "Bayesian Forecasting," Journal of the Royal Statistical Society, Ser. B, 38, 205-247. [263]

He, F., Yeung, L. F., and Brown, M. (2007), "Discrete-Time Model Representation for Biochemical Pathway Systems," IAENG International Journal of Computer Science, 34, 111-118. [271]

(2008), "Discrete-Time Model Representations for Biochemical Pathways," in Trends in Intelligent Systems and Computer Engineering, New York: Springer, pp. 255-271. [271]

Hürzeler, M., and Künsch, H. R. (1998), "Monte Carlo Approximations for General State-Space Models,” Journal of Computational and Graphical Statistics, 7, 175-193. [261,269]

Kitagawa, G. (1987), "Non-Gaussian State-Space Modeling of Nonstationary Time Series (C/R: P1041-1063; C/R: V83 P1231)," Journal of the American Statistical Association, 82, 1032-1041. [269-271]

(1996), "Monte Carlo Filter and Smoother for Non-Gaussian Non-Linear State Space Models," Journal of Computational and Graphical Statistics, 5, 1-25. [261]

Liu, J., and West, M. (2001), "Combined Parameter and State Estimation in Simulation-Based Filtering," in Sequential Monte Carlo Methods in Practice, eds. A. Doucet, J. De Freitas, and N. Gordon, New York: Springer-Verlag, pp. 197-217. [261] 
Nevins, J. (1998), "Toward an Understanding of the Functional Complexity of the E2F and Retinoblastoma Families," Cell Growth and Differentiation, 9, 585-593. [273]

Niemi, J. (2009), "Bayesian Analysis and Computational Methods for Dynamic Modeling," Ph.D. thesis, Duke University. [271]

Ravines, R. R., Migon, H. S., and Schmidt, A. M. (2007), “An Efficient Sampling Scheme for Dynamic Generalized Models,” Technical Report 201/2007, Departamento de Métodos Estatísticos, UFRJ. [261,267]

Rosenfeld, N., Young, J., Alon, U., Swain, P., and Elowitz, M. (2005), “Gene Regulation at the Single-Cell Level," Science, 307, 1962-1965. [261]

Sears, R., Ohtani, K., and Nevins, J. R. (1997), "Identification of Positively and Negatively Acting Elements Regulating Expression of the E2F2 Gene in Response to Cell Growth Signals," Molecular and Cellular Biology, 17, 5227-5235. [273]

Sorenson, H. W., and Alspach, D. L. (1971), "Recursive Bayesian Estimation Using Gaussian Sums," Automatica, 7, 465-479. [263]

Stroud, J. R., Müller, P., and Polson, N. G. (2003), "Nonlinear State-Space Models With State-Dependent Variances," Journal of the American Statistical Association, 98, 377-386. [261]

Tan, C., Song, H., Niemi, J., and You, L. (2007), "A Synthetic Biology Challenge: Making Cells Compute," Molecular BioSystems, 3, 343-353. [261]

Wang, Q., Niemi, J., Tan, C., You, L., and West, M. (2010), “Image Segmentation and Dynamic Lineage Analysis in Single-Cell Fluorescent Microscopy," Cytometry, Part A, 77, 101-110. [261]

West, M. (1992), "Modelling With Mixtures" (with discussion), in Bayesian Statistics 4, eds. J. Bernardo, J. Berger, A. Dawid, and A. Smith, Oxford: Clarendon Press, pp. 503-524. [261,263,277]

(1993a), “Approximating Posterior Distributions by Mixtures," Journal of the Royal Statistical Society, Ser. B, 54, 553-568. [263,277]

(1993b), "Mixture Models, Monte Carlo, Bayesian Updating and Dynamic Models," Computing Science and Statistics, 24, 325-333. [261,263,269,277]

West, M., and Harrison, J. (1997), Bayesian Forecasting and Dynamic Models (2nd ed.), New York: SpringerVerlag. [261-263,267,276]

Wilkinson, D. (2006), Stochastic Modelling for Systems Biology, London: Chapman \& Hall/CRC. [261,271] 